\title{
An Unusual Case of Critical Illness Polyneuromyopathy
}

Madhulika Mahashabde ${ }^{1}$, Gaurav Chaudhary ${ }^{2}$, Gangadharam Kanchi ${ }^{3}$, Shalesh Rohatgi ${ }^{4}$, Prajwal Rao ${ }^{5}$, Rahul Patil $^{6}$, Varun Nallamothu ${ }^{7}$

\begin{abstract}
Critical illness myopathy (CIM), critical illness polyneuropathy (CIP), and critical illness polyneuromyopathy (CIPNM) are the group of disorders that are commonly presented as neuromuscular weakness in intensive care unit (ICU) settings. They are responsible for prolonged ICU stay and failure to wean off from mechanical ventilation. We report a case of young female who was admitted with undiagnosed type I diabetes mellitus with diabetic ketoacidosis, severe hypokalemia, sepsis developed acute onset quadriplegia, and diaphragmatic palsy within 72 hours of ICU admission. On detailed investigation, CIPNM was diagnosed. In view of high morbidity, mortality, and poor prognosis, a guided approach to diagnose and treat in earliest possible duration might give better improvement and outcome of the illness. Despite all the odds, our patient showed good clinical improvement and finally got discharged.
\end{abstract}

Keywords: Diabetic Ketoacidosis, Intravenous immunoglobulin, Quadriplegia, Severe hypokalemia.

Indian Journal of Critical Care Medicine (2020): 10.5005/jp-journals-10071-23346

\section{INTRODUCTION}

Critical illness polyneuropathy (CIP), critical illness myopathy (CIM), and critical illness polyneuromyopathy (CIPNM) have similar presentation that cannot be differentiated clinically. They might be seen in patients suffering from severe sepsis, hyperglycemia, metabolic syndrome such as diabetic ketoacidosis, severe electrolyte imbalances, multisystem organ failure, and patients who have been treated with neuromuscular blocking agents and large doses of corticosteroids. The symptoms may present as early as 72 hours of intensive care unit (ICU) admission. ${ }^{\text {? }}$

This case report highlights the diagnosis and management approach to the patient who develops CIPNM.

\section{Case Description}

A 27-year-old female was admitted with 2 days history of fever, abdomen pain, and three episodes of vomiting with severe dehydration. She was in altered sensorium, and her vitals were otherwise stable. On examination, no obvious abnormality was seen. On preliminary investigations, plasma blood sugar was high. Arterial blood gas analysis showed severe metabolic acidosis $\left(\mathrm{pH}: 6.95, \mathrm{PCO}_{2}: 15\right.$, and $\left.\mathrm{HCO}_{3}{ }^{-}: 6\right)$. Routine investigations were unremarkable except for severe hypokalemia $\left(\mathrm{K}^{+}: 1.9\right)$, and urine ketone bodies were large, sugar: $3+$. There was no significant past history, no significant family history, and no addictions.

On second day, she started developing acute onset flaccid paralysis in all four limbs, symmetrical, proximal more than distal. On detailed examination, power was $1 / 5$ in both upper limbs, $0 / 5$ in both lower limbs, all deep tendon reflexes were diminished, and bilateral plantars were mute. Cranial nerves were intact, with no sensory loss. After 4-5 hours, she developed paradoxical breathing, not maintaining saturation in room air. We intubated her immediately and kept her on mechanical ventilation. Despite the correction of acidosis and large potassium deficits, her weakness continued to persist. On subsequent days, we were not able to wean her off from assisted ventilation. Then, we investigate further to evaluate acute onset quadriplegia. Neurophysician opinion was taken, and nerve conduction velocity (NCV) and electromyography
1-3,6,7Department of General Medicine, Dr DY Patil Medical College, Pune, Maharashtra, India

4,5Department of Neurology, Dr DY Patil Medical College, Pune, Maharashtra, India

Corresponding Author: Gangadharam Kanchi, Department of General Medicine, Dr DY Patil Medical College, Pune, Maharashtra, India, Phone: +918801999535, e-mail: gangadharamkanchi@hotmail.com

How to cite this article: Mahashabde M, Chaudhary G, Kanchi G, Rohatgi S, Rao P, Patil R, et al. An Unusual Case of Critical Illness Polyneuromyopathy. Indian J Crit Care Med 2020;24(2):133-135.

Source of support: Nil

Conflict of interest: None

(EMG) studies revealed primary muscle disease with axonal polyneuropathy (Fig. 1).

On further investigations, $\mathrm{HBA}_{1} \mathrm{C}$ was $7.5 \%$, GAD antibodies were positive, CPK total increased to $1171 \mathrm{U} / \mathrm{L}$, blood cultures isolated revealed coagulase-negative Staphylococcus, urine culture isolates budding yeast cells, cerebrospinal fluid examination was within normal limit, and magnetic resonance imaging brain revealed diffuse cerebral edema (Fig. 2). In view of prolonged mechanical ventilation, tracheostomy was done on day 18. Later on, she developed ventilator-associated pneumonia, and chest roentgenogram showed nonhomogeneous patches with ground glass appearance in both lower zones. High-resolution computed tomography of chest suggested bilateral infiltration of lung fields with ground glass appearance most likely pneumonia. Sputum culture was positive for Klebsiella pneumoniae.

Finally, we made a diagnosis of type 1 diabetes mellitus with diabetic ketoacidosis, sepsis, severe hypokalemia, and CIPNM. Hyperglycemia was controlled, and diabetic ketoacidosis was corrected as per the protocol. Pneumonia and sepsis were treated with antibiotics according to the culture reports, and large potassium deficits were corrected with $\mathrm{KCl}$, almost requiring $300 \mathrm{mEq}$./day. In the context of CIPNM, we decided to give intravenous immunoglobulins (IVlg) at $1 \mathrm{~g} / \mathrm{kg}$ in divided doses

() The Author(s). 2020 Open Access This article is distributed under the terms of the Creative Commons Attribution 4.0 International License (https://creativecommons. org/licenses/by-nc/4.0/), which permits unrestricted use, distribution, and non-commercial reproduction in any medium, provided you give appropriate credit to the original author(s) and the source, provide a link to the Creative Commons license, and indicate if changes were made. The Creative Commons Public Domain Dedication waiver (http://creativecommons.org/publicdomain/zero/1.0/) applies to the data made available in this article, unless otherwise stated. 

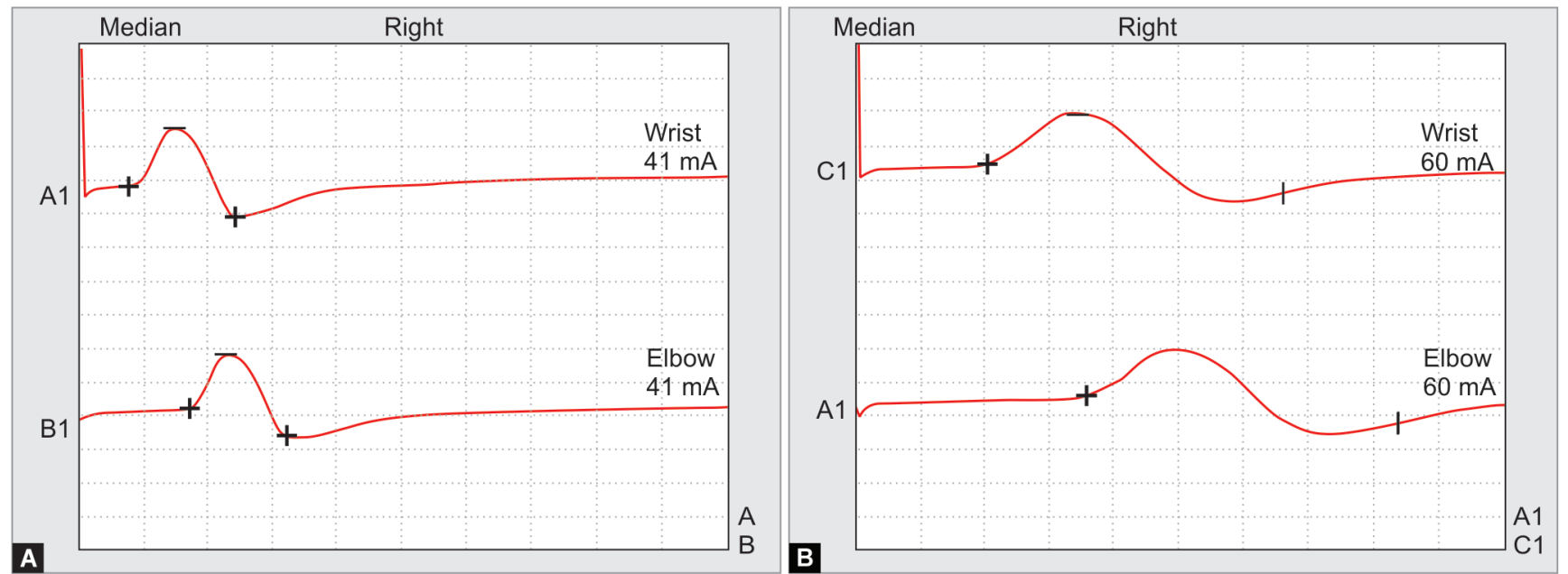

Figs $1 \mathrm{~A}$ and B: Nerve conduction velocity studies: (A) On admission; (B) 3 weeks later. Note, there is a marked decline in amplitude and increase in duration

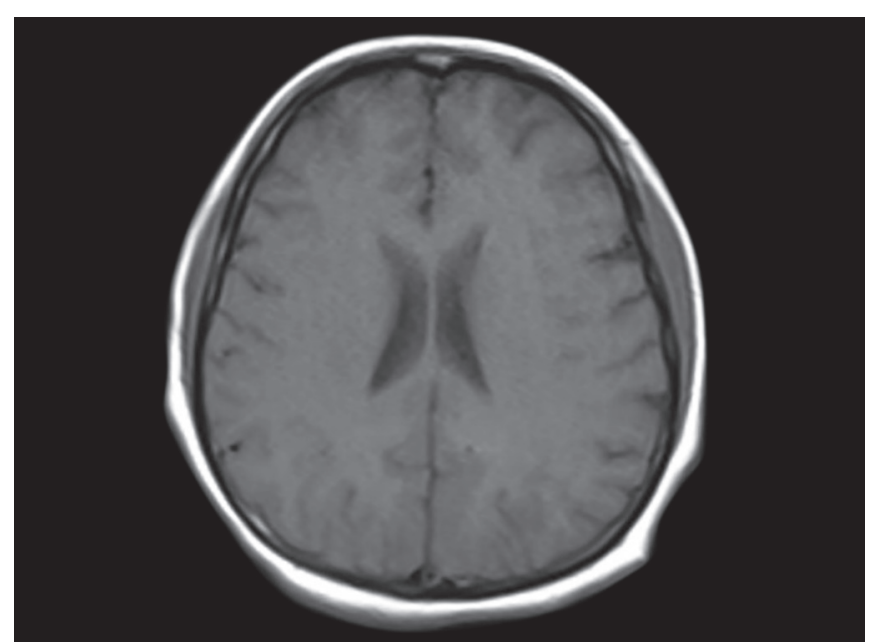

Fig. 2: MRI of brain image showing diffuse cerebral edema

for 5 days. ${ }^{2}$ Parenteral nutritional support, antioxidant therapy, and physiotherapy were given accordingly.

Later on, she was improved clinically, power was regained, and reflexes were present. We weaned off mechanical ventilation. Repeat electrophysiological (NCV and EMG) studies suggested the recovery phase of polyneuromyopathy, all other laboratory tests were improved, and she was discharged after 45 days of hospital stay with grade $4 / 5$ power in all the limbs. She was advised to continue regular physiotherapy and insulin analogs.

\section{Discussion}

Critical illness polyneuropathy, CIM, and CIPNM are often referred to as ICU-acquired weakness (ICUAW). There are no probable etiological factors other than critical illness. The probable risk factors include severe sepsis, hyperglycemia, severe electrolyte imbalances, metabolic syndrome, increased duration of multiorgan failure, hyperosmolality, parenteral nutrition, renal replacement therapy, vasopressors, corticosteroids, neuromuscular blocking agents, and aminoglycosides ${ }^{1,3}$ (Flowchart 1).

Clinical suspicion is aroused when there is a failure to wean off from mechanical ventilation, despite the improvement in underlying critical illness. Muscle wasting is variable, and the
Flowchart 1: ICU-acquired weakness association with critical illness

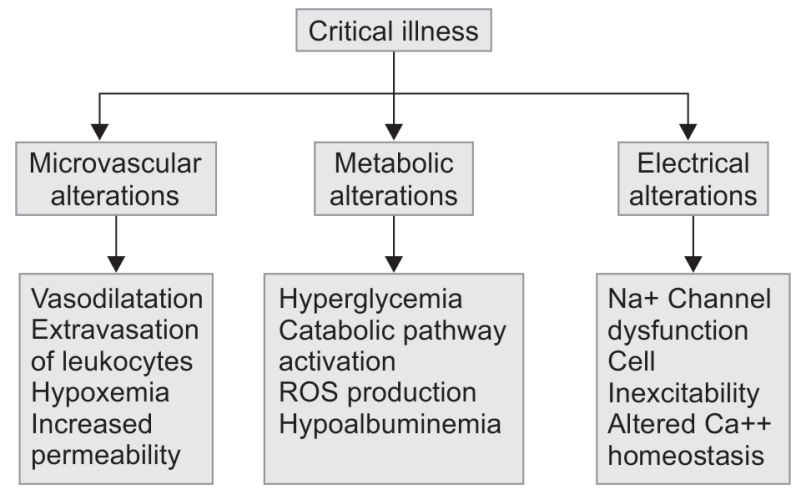

sensory system may or may not be affected. Autonomic nervous system is preserved. ${ }^{2}$

The precise mechanism of developing CIPNM is unclear, but circulating factors include cytokines associated with sepsis, and multiorgan failure is thought to play a key role (Tables 1 to 4). It has been reported that $70 \%$ of the patients with sepsis syndrome have certain degree of neuropathy and severe respiratory muscle weakness requiring prolonged ventilation resulting in failure to wean off. There might be muscle wasting due to the loss of thick myosin filaments. ${ }^{4}$ However, the process may take place for many days and needs tracheostomy for prolonged mechanical ventilation. Some patients have residual long-term weakness with atrophy and muscle fatigue, which was not present in our patient.

\section{Clinical Significance}

In summary, CIP, CIM, and CIPNM are commonly seen as neuromuscular weakness in patients admitted in ICU. In total, $45 \%$ of the patients who were diagnosed with ICUAW will die within their hospital admission and further $20 \%$ will die in an year after discharge. ${ }^{3}$ Once the diagnosis of ICUAW has been established, it is advisable to initiate the management as early as possible in early stages. Future management strategies should target the proinflammatory cytokines, free radical pathways, controlling the other risk factors that include hyperglycemia, electrolyte imbalances, and sepsis. According to the analysis in the study by Mohr et al., ${ }^{5}$ 
Table 1: Differential diagnoses of failure to wean from mechanical ventilation

\begin{tabular}{ll}
\hline Motor neuron & Critical illness polyneuropathy \\
& Critical illness polyneuropathy/ \\
myopathy & Amyotrophic lateral sclerosis \\
& Heavy metal toxicity \\
& Guillain-Barré syndrome \\
& Poliomyelitis vasculitis \\
& Neuromuscular blockade \\
& Myasthenia gravis \\
Neuromuscular junction & Lambert-Eaton myasthenic \\
& syndrome \\
& Botulinum toxicity \\
& Critical illness myopathy \\
& Mitochondrial myopathy \\
Muscle & Muscular dystrophy (e.g., \\
& myotonic dystrophy) \\
\hline
\end{tabular}

Adapted from Shepherd et al. ${ }^{1}$

Table 2: Diagnostic criteria of critical illness polyneuropathy

1. The patient is critically ill patient (sepsis and multiple organ failure)

2. Difficulty to wean off ventilator after non-neuromuscular causes such as heart and lung disease have been excluded

3. Limb weakness

4. Electrophysiological (nerve conduction velocity) evidence of axonal motor and sensory polyneuropathies

Adapted from Bolton ${ }^{4}$

early administration of IVIg might improve and mitigate the CIPNM. However, many trails have been going on to establish the role of IVIg in CIP/CIM.

Despite high morbidity and mortality, our patient showed clinical improvement in her early phase of the hospital stay. She got discharged with minimal post illness residual weakness with almost complete recovery. In conclusion, maintaining high-level suspicion of critical illness-associated weakness in those patients admitted in ICU, early diagnosis, control of the risk factors, and the
Table 3: Diagnostic criteria of critical illness myopathy

1. SNAP amplitudes $>80 \%$ of the lower limit of normal

2. EMG studies with short-duration, low-amplitude MUPs with early or normal full recruitment, with or without fibrillation potentials

3. Absence of a decremental response on repetitive nerve stimulation

4. Muscle histopathological findings of myopathy with myosin loss

5. CMAP amplitudes $<80 \%$ of the lower limit of normal in two or more nerves without conduction block

6. Elevated serum creatine kinase

7. Demonstration of muscle in-excitability

Adapted from Bolton ${ }^{4}$

SNAP, sensory nerve action potential; EMG, electromyography; MUP, motor unit potential; CMAP, compound muscle action potential

Table 4: Diagnostic criteria of critical illness polyneuromyopathy

1. Patient admitted in intensive care unit

2. Patient meets the criteria of critical illness polyneuropathy

3. Patient meets the criteria of critical illness myopathy

Adapted from Appleton ${ }^{3}$

treatment given accordingly at the earliest possible duration might reduce the morbidity and mortality related to CIP/CIM.

\section{References}

1. Shepherd S, Batra A, Lerner DP. Review of critical illness myopathy and neuropathy. Neurohospitalist 2017;7(1):41-48. DOI: $10.1177 / 1941874416663279$.

2. Zhou C, Wu L, Ni F, Ji W, Wu J, Zhang H. Critical illness polyneuropathy and myopathy: a systematic review. Neural regeneration research 2014;9(1):101-110. DOI: 10.4103/1673-5374.125337.

3. Appleton R, Kinsella J. Intensive care unit-acquired weakness. Continuing education in anaesthesia, critical care and pain 2012;12(2):62-66. DOI: 10.1093/bjaceaccp/mkr057.

4. Bolton CF. Neuromuscular manifestations of critical illness. Muscle Nerve 2005;32(2):140-163. DOI: 10.1002/mus.20304.

5. Mohr M, Englisch L, Roth A, Burchardi H, Zielmann S. Effects of early treatment with immunoglobulin on critical illness polyneuropathy following multiple organ failure and gram-negative sepsis. Intensive Care Med 1997;23(11):1144-1149. DOI: 10.1007/s001340050471. 